\title{
Challenging the clinically-situated emotion-deficient version of empathy within medicine and medical education research
}

\author{
Barret Michalec ${ }^{1} \cdot$ Frederic W. Hafferty ${ }^{2}$
}

Accepted: 28 September 2021 / Published online: 22 November 2021

(c) The Author(s), under exclusive licence to Springer Nature Limited 2021

\begin{abstract}
In this paper, we argue that the notion of a clinically-situated empathy (e.g. physician empathy), is potentially problematic as it perpetuates an emotion-deficient version of empathy within medicine and medicine education research. Utilizing classic and contemporary empathy theory from various social science disciplines, we discuss how empathy in the general sense differs conceptually from clinically-situated empathy_paying particular attention to the role of emotional contagion. To highlight this contrast, we draw upon Hojat et al.'s model of physician empathy and how this body of work reflects broader medical-cultural norms that problematize the role and impact of emotions within the clinical encounter. Alternatively, we present a more encompassing model of empathy drawing upon the fields of social-psychology and social-neuroscience in order to bring the notion of "feeling with" and emotional contagion more specifically, into medical education, medical education research, and medicine more generally.
\end{abstract}

Keywords Empathy $\cdot$ Emotional Contagion $\cdot$ Medicine $\cdot$ Medical Education

\section{Introduction}

From the early teachings of Osler (1906) featured in "Aequanimitas", to Blumgart's (1964) "neutral empathy", to Lief and Fox's (1963) notion of "detached concern", the culture and practice of medicine has consistently promoted and promulgated the essentiality for physicians (and medical students) to maintain an emotionally detached and physiologically refrained position within the clinical encounter-holding both the patients' and their own emotions at arms-length so as not to share, and

Barret Michalec

barret.michalec@asu.edu

1 Arizona State University, Phoenix, USA

2 Mayo Clinic, Rochester, USA

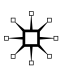


in turn be encumbered by, affective states that would undoubtedly cloud objectivity and clinical reasoning.

Within more contemporary empathy-related research, Hojat et al.'s (2009) conceptualization and operationalization of "physician empathy" has gained prominence and popularity (Sulzer et al. 2016). Relatively devoid of emotional aspects of the empathy experience (specifically emotional contagion), and with explicit focus on cognitive and behavioral skills, this version of a clinically-situated empathy has become entrenched within medicine and medical education research. However, this version of empathy does not align with broader understandings of the empathy concept from fields such as social-psychology and social-neuroscience. And although this lack of alignment has been hinted in previous literature (Sulzer et al. 2016; Preusche and Lamm 2016; Finset and Ømes 2017), it has yet to be fully dissected. Furthermore, there has been minimal attention to the potential deleterious effect(s) of the dominance of and reliance on a cognitive- and behavioral-specific version of empathy within medicine and medical education research.

In this paper, utilizing classic and contemporary empathy theory and highlighting related social theoretical frameworks, we challenge the concept of physician empathy as well as the broader notion of a clinically-situated version of empathy, and argue that the apparent embeddedness of a cognitive-specific, emotion-deficient concept of empathy within medicine and medical education, specifically in the arena of research and scholarship, may have significant negative impacts on students' and professionals' socioemotional well-being and humanism. Through the examination of overarching themes and roles of emotions and emotionality within medicine, we show how the rise of an emotion-deficient empathy is reflective of a (misguided yet) persistent focus on clinical knowledge, detachment, and the rationality of science (Michalec 2012; Shapiro 2011; Underman and Hirshfield 2016). We conclude by discussing the potential educational and professionalization benefits of accepting the emotional components of the empathy experience (specifically emotional contagion) in tandem with the cognitive. In this context, we suggest instituting a broader conceptualization and understanding of empathy within medical education and practice that includes key socio-emotional and cognitive aspects in order to possibly unlock innate human capacities and skills that may have been suppressed by overarching cultural norms and practices.

\section{Classic and contemporary empathy theory}

Empathy, in the general vernacular, is an emotional and cognitive experience of another's emotional state. Historically, the term comes the German word Einfühlung, the notion of feeling in(to), to project oneself into an object that they observe (Wispè, 1987; Jahoda 2005, Davis 1994; Lanzioni 2018). Theodor Lipps, a German philosopher, introduced Einfühlung to the field of psychological in the early 1900s, where it quickly was met with debate and cross-conceptualization (namely with sympathy - a more prominent term at that time). Lipps is credited with employing the term not just in regards to aesthetics (art, objects, etc.) which is where the term originated, but with how people interact as well. In fact, Lipps argued that 
Einfühlung was instinctual and that this feeling into was automatically triggered by observing other's emotional expressions (Montag and Heinz 2008). Although a number of early twentieth century scholars wrestled with various tenets of Einfühlung, it was Edward Tichener, an American psychologist, who teased the concept from sympathy and translated Einfühlung to "empathy" as the process of reading or feeling ourselves into objects (Jahoda 2005).

Since Tichener's work, the term has continued to evolve through scholarly discussion and exploration in the fields of psychology, sociology, neuroscience, and philosophy (and sub-fields therein) (Lanzoni 2018; Ruiz-Junco 2017; McCaffree 2020). Slight differences notwithstanding, there is consensus among prominent empathy theories that empathy is a social experience in that it is other-oriented and stems from the recognition of another individuals' emotional state (Batson et al. 2005; Eisenberg et al. 1994; Davis 1994; Dovidio et al. 1990). The observer evaluates particular cues from the expression of emotion (verbal and/or nonverbal) and, through shared meanings with their interaction partner, recognizes that the other is experiencing a particular emotional state (Eisenberg et al. 2006). Such recognition, automatically triggers a similar physio-emotional response in the observer (i.e. emotional contagion), and, in turn, directly stimulates the cognitive and affective processes and mechanisms nested within the experience of empathy related to perspective taking, self- and other-awareness, imagining, emotional regulation, affective reactivity, mental flexibility, empathic concern, among others. (Hoffman 1981; Hoffman 2000; Roberts and Strayer 1996; Decety and Jackson 2004; de Waal 2009). Whether empathy is primarily emotional or cognitive, or an additive feature of both, is still an active debate. Regardless, there is common agreement among social-psychological and social-neuroscience scholars that there are both cognitive and affective processes at play in the empathy experience (Eisenberg and Strayer 1987; Wispé 1991 Decety and Jackson 2004; Davis 1994; Hoffman 2000; Roberts and Strayer 1996; Eisenberg et al. 1994; Ickes 2003; Preston and de Waal 2002; Feshbach and Feshbach 2009).

Notably, within this broader conceptual discussion, there is a continued effort to disentangle empathy and sympathy. Among (fairly) contemporary scholars, there appears to be consensus that sympathy does not involve feeling the same or similar feeling as another person-a key element of the empathy experience. Rather, sympathy is a heightened sensibility to the other person's negative emotional state and an accompanying motivation (i.e. empathic concern, sorrow) to alleviate that negative state through help, comfort, or support (Wispé 1991; Eisenberg 2006; Decety and Moriguchi 2007; Preusche and Lamm 2016). Within this framework, we promote the commonly accepted differentiation: whereas empathy involves a "feeling with", sympathy involves a "feeling for".

\section{The role of emotional contagion}

The social dynamic of feeling with is paramount to the empathy experience, and within this interpersonal phenomena there is an unfolding cascade of initial implicit understanding, mimicry, and affective shared experience that broadly can 
be understood as emotional contagion. Put simply, the term "emotional contagion" captures the idea that emotions are contagious, that you can involuntarily catch them from others (like a cold), and is innate and fundamental to the empathy experience (Hatfield et al. 1994; Smither 1977; Decety and Jackson 2004; Trevarthen and Aitken 2001; Preusche and Lamm 2016; Singer and Lamm 2009; Preston and de Waal 2002; de Waal 2008).

Hatfield et al. (1993) developed a social theory of emotional contagion and defined phenomenon as "..the tendency to automatically mimic and synchronize expressions, vocalizations, postures, and movement with those of another person's and, consequently, to converge emotionally." Over time, emotional contagion has taken on significant conceptual breath (Prochazkova and Kret 2017; Preston and de Waal 2002; Gonzalez-Liencres et al. 2014; Meyza and Knapska 2018). Moreover, empathy scholars from various fields have argued that not only is emotional contagion innate and involuntary, but that the shared experience has evolutionary resiliency as it fosters interpersonal connections, social cohesiveness, solidarity, and expedites communication and therefore sustains and advances dyads, groups, and even species (Trevarthen 1979; Eisenberg and Strayer 1987; Hatfield et al. 1993; Hoffman 2000; Decety and Jackson 2004; de Vignemont and Singer 2006).

A descriptive example of how emotional contagion is nested within the experience of empathy is most clearly presented by de Waal's Russian Doll Model (2008; Preston and de Waal 2002; de Waal and Preston 2017). As shown in Fig. 1, the Russian Doll Model of empathy argues that at the core of the empathy experience are simple hard-wired mechanisms such as emotional contagion, whereas the more complex, higher cognitive level functioning and abilities (i.e. empathic concern, perspective taking, self-other awareness) are at its outer layers (de Waal 2012; de Waal and Preston 2017). For de Waal, there is no value in separating the cognitive aspects of empathy from the emotional aspects of empathy, doing so only loses sight of the integrated whole experience of empathy. Moreover, de Waal's approach highlights emotional contagion as an innate, core element of the empathy experience (de Waal 2008, 2012).

Having provided a general description of the primary tenets of the empathy experience from the social-psychological and social-neuroscience perspectives, showcasing the role of emotional contagion, we now briefly outline how the term is primarily conceptualized and operationalized within contemporary medicine and medical education research and commentary.

\section{Enter "physician empathy"}

As noted above, prominent models exploring and explaining effective and efficient degrees of interpersonal connectivity between doctors and patients in the clinical encounter advocate for detachment and emotional distance (Halpern 2001). In the early 2000s, there was an eruption of empathy research and commentaries in medical and medical education journals (Pedersen 2009; Decety and Fotopoulou 2015), with a continued embrace by medicine and medical education of an emotional-deficient 


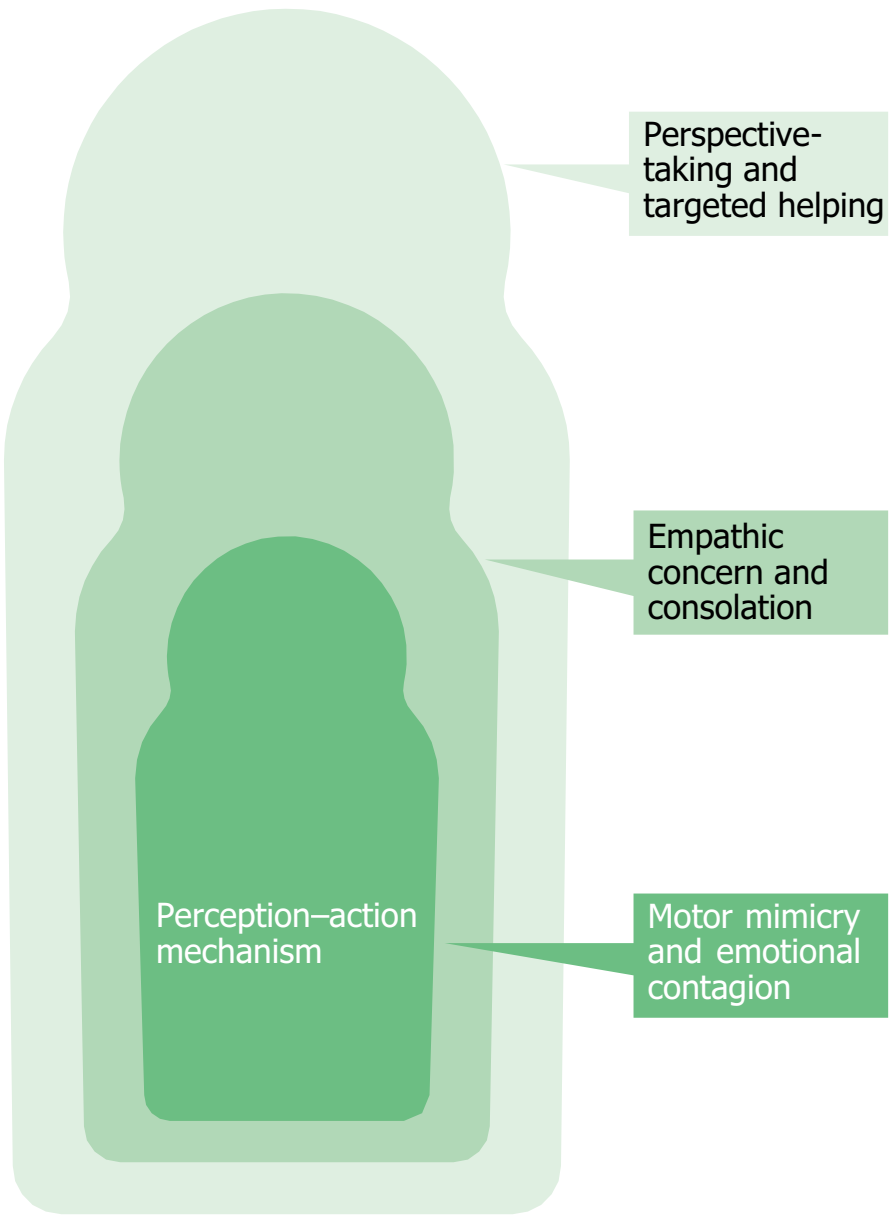

Fig. 1 The Russian-doll model of the evolution of empathy. Various components of the empathic response, which have been added layer upon layer during evolution, remain functionally integrated. At its core is the perception-action mechanism, which induces a similar emotional state in the observer as in the target. Its most basic expressions are motor mimicry and emotional contagion. The doll's outer layers, such as empathic concern and perspective-taking, build upon this core socio-affective basis while increasingly requiring emotion regulation, self-other distinction and cognition. Even though the doll's outer layers depend on prefrontal functioning, they remain fundamentally linked to the core perceptionaction mechanism. Adapted with permission from: de Waal (2003)

version of empathy specific to the clinical encounter, and that is posed as general empathy.

During this time, and as a prominent example of this eruption, Hojat et al. (2001, 2002a, 2002b, 2003) formulate a conceptualization of empathy that is specific to interactions the clinical realm (i.e. doctor and patient) — a "physician empathy" and that focuses almost exclusively on the cognitive components of empathy. Although Hojat et al.'s conceptualization is presented in research prior to 2009, we offer what we believe to the most descriptive definition physician empathy from their landmark 
study on medical students' loss of empathy, "The Devil is in the Third Year" (Hojat et al. 2009):

"..we define empathy in the context of medical education and patient care as a predominantly cognitive (as opposed to affective or emotional) attribute that involves an understanding (as opposed to feeling) of patients' experiences, concerns, and perspectives combined with the capability to communicate this understanding. An intention to help by preventing and alleviating pain and suffering is an additional feature of empathy in the context of patient care" (Italics added by original authors) (p. 1183)

In their conceptualization of physician empathy, Hojat et al. unmistakably highlight the cognitive components of empathy-even placing the emotional components in opposition to the cognitive. Moreover, there is an accompanying emphasis on a behavioral component to the empathy experience. Physicianspecific empathy, according to Hojat (2007), is an intellectual attribute rooted in understanding and reasoning that is explicitly distinguished from emotion sharing and self-other emotion processing. In fact, Hojat et al. (2001) appears to corral the emotional aspects of empathy into the completely separate concept of sympathy while advancing a long-standing cultural belief within medicine that any form of emotion sharing can significantly interfere with clinical objectivity in diagnosis and treatment.

"In a medical context, empathy can be viewed as an uncritical understanding of a patient's inner feelings and experiences as a separate individual, as opposed to 'feeling with' the patient, which characterizes sympathy. Therefore, it is important to note that empathy, as opposed to sympathy, is defined mostly a cognitive rather than affective mental process." (p. 352)

Through their work, Hojat and colleagues sought to establish a clear, consistent definition of what to study regarding empathy in professional medicine and medical education realms. Moreover, by providing an accompanying tool to assess, the Jefferson Scale of Physician Empathy (JSPE) (Hojat et al. 2001), the team galvanized scholars in the arena of how to study this particular form of empathy. Because the assessment tool stems from their conceptualization of physician empathy, the operationalization of the term is also primarily focused on cognitive and behavioral components (Hojat et al. 2001; Hojat 2007). Moreover, although Hojat et al.'s initial work stressed "physician empathy"-this scale's title was later condensed to simply the Jefferson Scale of Empathy (Hojat et al. 2018). This shift in the title of the tool (i.e. the dropping of term "physician") appears to coincide with efforts to expand the tool's intended audience to include a broader range of health profession students and professionals. This change in title notwithstanding, Hojat et al. continue to maintain the exact same conceptualization of a clinically-situated empathy as referenced above (Hojat et al. 2020) as they consistently argue that this conceptualization is specific to empathy in the clinical context- “...to capture the essence of empathy in the context of health professions education and patient care" (Hojat et al. 2018, p. 901). Within this current paper, we argue that this clinically-situated version of 
empathy is not conceptually or operationally synonymous with the general empathy concept. Finally, for the sake of clarity and to maintain distinction between the clinically-situated empathy concept and the general empathy concept, we will refer to Hojat et al.'s version of empathy within this paper as "physician empathy" and refer to their particular scale as the "JSPE."

There have been sporadic reminders from empathy scholars that Hojat et al.'s physician empathy (and the JSPE) has become conceptually estranged from general empathy (Sulzer et al. 2016; Preusche and Lamm 2016; Finset and Ømes 2017). A principle focus of this estrangement is the formulation of a clinically-situated empathy that is shorn of emotional contagion. Although emotional contagion is viewed by classic and contemporary empathy scholars (as discussed earlier) as an essential, innate ingredient in the empathy experience, emotional contagion is not only missing from Hojat et al.'s physician empathy, but is explicitly rejected. Another distancing feature is Hojat and colleagues' behavioral framing to physician empathy. Classic and contemporary empathy scholars state that although the experience of empathy may facilitate helping behaviors such as altruism, compassion, and social support, it does not necessitate helping behavior or supportive communication (Cialdini et al. 1987; Batson 1991; Smith et al. 1989; Decety and Jackson 2004; Decety and Moriguchi 2007; Bergman Blix 2019). These empathy scholars consistently state that various intra- and interpersonal, situational, contextual, and motivational factors and cues will impact if and to what extent the experience of empathy might translate into behavior. Yet, this behavioral component is not only assumed but consistently marquee within physician empathy.

Despite these clear conceptual dissimilarities, within the medical and medical education literature, physician empathy has not only gained scholastic popularity, but has also mistakenly become synonymous with empathy in the general sense, as the terms are consistently utilized interchangeable within the literature as noted above and further exemplified by the shifting of the JSPE to simply the JSE- - suggesting and promulgating their (erroneous) synchronicity.

\section{Physician empathy and clinical empathy}

Although Hojat et al.'s "physician empathy" is more prominent, the term "clinical empathy" (see Halpern 2001, 2003, 2014) is also featured to denote the empathy experienced by physicians or medical students with patients and/or the patients' family within contemporary medical and medical education literature. Given their synchronous presence in the literature, it is important to briefly outline key conceptual similarities and differences.

Halpern's (2014) model of clinical empathy promotes perception, curiosity, nonverbal attentiveness, proportional concern for the patient, and self-awareness. It is through the development and presentation of these clinician-based qualities that invites patients to provide personal and emotional details, which in turn, allows the clinician to imagine how the patient's emotional experience, to resonate nonverbally and feel an appropriate level of concern towards the patient (Halpern 2014). 
Unlike Hojat et al. Halpern explicitly champions the value and role(s) of emotions in empathy and the clinical setting. Yet, and somewhat similar to Hojat et al. Halpern $(2003,2014)$ urges that the clinician not vicariously experience the patient's emotions - that this would be "absurdly demanding". Rather, according to Halpern, physicians should utilize emotional attunement and, in turn, imagine what the patient may be experiencing emotionally. Moreover, despite arguing for the presence and value of emotionality in the clinical encounter, for Halpern (2003) the elements of clinical empathy are "..special professional skills...distinguished by the use of this subjective experiential input for specific, cognitive aims."

In sum, although presenting nuanced variations, both clinical empathy and physician empathy, at root, are cognitively-prominent versions of empathy that warn against the sharing of emotional states. Although Halpern's clinical empathy was/ is not completely overshadowed by Hojat's physician empathy, the availability of a concise and valid tool to assess a version of clinically-situated empathy essentially establishes physician empathy as the go-to empathy concept-of-choice for empirical studies-propagating and promoting a cognition-focused and emotion-deficient empathy experience in the clinical realm.

\section{The embeddedness of an emotion-deficient empathy}

\section{The prominent presence of physician empathy in medical education research}

A recent systematic review of evaluation-based studies of empathy training programs in medical education (Batt-Rawden et al. 2013) found that $60 \%$ of those studies with validated outcome measures used the JSPE-with the JSPE used in three times as many studies as the other empathy scales examined. In another systematic review on the conceptualization and assessment of empathy within medical education, Sulzer et al. (2016) show the studies they reviewed featured cognitive, emotional, and/or behavioral framings of empathy, and that $85 \%$ of those articles providing a definition of empathy presented empathy as either in-part or entirely composed of cognitive/thinking processes - and with a majority of these utilizing Hojat's definition of physician empathy. Furthermore, whereas cognitive/thinking processes were the most prevalent components in those definitions, affective/feeling processes were the least prevalent definitional components. According to the authors, roughly $25 \%$ of all definitions of empathy featured a feeling dimension, "...and some quite specifically excluded it...". Furthermore, of the empirical studies in their sample (with empirical studies on empathy are outnumbered by commentaries), $41 \%$ of the studies (majority) employed some version of the JSPE.

Given its central presence within Sulzer et al.'s review, as well as its dominating citation count within medical education literature, Hojat et al.'s (2009) The Devil is in the Third Year: A Longitudinal Study of Erosion of Empathy in Medical School is clearly a landmark work featuring the notion of physician empathy. A quick examination of the impact of this specific article via Google Scholar (searches conducted 6/4/20) shows that the article has been cited over 1310 times since its debut 
in 2009-for simplicity-sake we can say that it has been cited roughly 120 times a year. In comparison, conducting the same rudimentary examination of another seminal piece in medical education, Hafferty and Franks' (1994) The Hidden Curriculum, Ethics Teaching, and the Structure of Medical Education, which showcases the hidden curriculum concept, has been cited over 1630 times since its 1994 debut -roughly 62 times a year, which is almost half as often as the Hojat et al. paper.

\section{Medical and medical education culture as a prime breeding ground for physician empathy}

Taken together, these data showcase the embeddedness of Hojat et al.'s emotiondeficient conceptualization and operationalization of physician empathy within medicine and medical education research. Yet beyond the entrenchment within the literature, this emotion-deficient version of empathy is also reflective (and/or perhaps a by-product) of an overarching culture within medicine and medical education that problematizes emotions and emotionality (McNaughton 2013). Even Halpern (2001, 2003) acknowledges that there is a persistent perspective within medicine that emotions are "unscientific" and "interfere with objectivity" despite empirical research consistently presenting evidence to the contrary.

This depiction of medicine and medical education as emotion-deficient institutions is perhaps best presented by Dr. Ofri's (2012) OpEd in the New York Times,

"By now, even the most hard-core, old-school doctors recognize that emotions are present in medicine at every level, but the consideration of them rarely makes it into medical school curriculums, let alone professional charters. Typically, feelings are lumped into the catch-all of stress or fatigue, with the unspoken assumption that with enough gumption these irritants can be corralled."

Within medicine in general, and in clinically-situated empathy-related discourse more specifically, there is a consistent depiction of emotions as not only troublesome and unruly, but also as mechanisms by which doctors and medical students could lose themselves through overt vulnerability (Shapiro 2011). As Preusche and Lamm (2016) state, "Another major advantage of emphasis on the cognitive basis of empathy for [medical education] is the implication that the physician is in control of his/her empathy." In the clinically-situated version of empathy, the physician is active, in control of their thoughts (and feelings). Yet, in the general, lay-person empathy experience there are involuntary, automatic, even passive, emotion-specific processes such as emotional contagion. Although an essential element of the empathy experience within the fields of social-psychology and social-neuroscience, the innate, involuntary, automatic phenomenon of emotional contagion is framed as dangerous and dysfunctional for physicians and medical students in their clinical encounter. 


\section{Why the need for a clinically-situated empathy like physician empathy?}

Recent research has indicated that the empathy experience is the default response/ reaction (i.e. engaged automatically) when witnessing another in distress or negative affect (McAuliffe et al. 2020). And we know from classic (and contemporary) empathy theory, that the experience of empathy requires a degree of openness and sensitivity (even vulnerability) to the emotional state of the other. Because of their primary responsibilities, physicians, regardless of specialty, continuously observe and confront pain and suffering - therefore, consistently empathizing with patient after patient is seen as painful toll on psychological and emotional resources, which could lead to compassion fatigue, emotional exhaustion, and burnout (Gleichgerrcht and Decety 2012; Decety et al. 2010; Hojat et al. 2009). The predominant doctrine within medicine has been that because of the exposures to and bombardment of others' negative states, physicians must regulate their other-orientation, own emotional experiences, and general sensitivity to patients' emotions. Relatedly, even the fundamental mechanism of connecting with and sharing patients' emotions (i.e. emotional contagion) is perceived as highly deleterious-which is even stated explicitly by Hojat and Halpern (as noted above). Given that the overarching culture of medicine is persistently dismissive of emotions and emotionality, the need to regulate the aspects of empathy experience that are specific to emotional sharing and emotional contagion creates the utility and necessity of an emotion-deficient clinically-situated version empathy.

We have provided the evidence that this clinically-situated version of empathy (e.g. Hojat et al.'s physician empathy) is conceptually distinct from general empathy. In their introduction of the JSPE, Hojat et al. (2001), show corroboration between the JSPE and instruments that measure general empathy (e.g. Davis's IRI, Mehrabian's BEES) - suggesting that physician empathy is a sibling to empathy, rather than a distant third cousin. Yet, in reference to the IRI and BEES, Hojat et al. state, "It is important to note that these instruments have been developed for the general population, and none is specific to patient care situations." Therefore, whereas there may be some conceptual overlap, even Hojat et al. suggest there is distinction between physician empathy and empathy experienced among the "general population." With this additional operational distinction in mind, we suggest that physician empathy and the broader notion of a clinical-situated empathy, is not equitable to the general empathy experience, but rather a set of professionally developed (primarily) cognitive and behavioral skills (Gleichgerrcht and Decety 2012; Halpern 2003; Hojat 2007) that can be explicitly taught and assessed.

\section{Emotional labor and potential consequences of an emotion-deficient clinically-situated empathy}

Larson and Yao (2005), and more recently Vinson and Underman (2020), have argued that the practice of a clinically-situated empathy is a form of emotional labor in the patient-physician relationship and within medical training. Emotional labor is the act(s) of displaying socially desirable emotions in/during interpersonal 
interactions where a worker (e.g. physician, medical student) is providing service to a customer (e.g. patient) (Hochschild 1983). During these interactions, the worker may attempt to regulate their emotion states and related emotional displays in order to be more in-line with work-appropriate emotional displays in that specific context (Wharton 2009). Emotional labor can have significant adverse effects on workers' psychological and emotional resources. In turn, persistent engagement with emotional labor can lend to emotional exhaustion, compassion fatigue, and burnout (Brotheridge and Lee 2002; Vinson and Underman 2020). Larson and Yao (2005) suggest that in order to meet patients' expectations of empathic treatment that physicians engage not only in "surface acting" (i.e. "faking" emotional displays by forcibly manipulating their face, posture, and/or voice), but also in "deep acting", or changing their perceptions of the situation or altering their emotional focus in order to modify their automatic emotional reactions and their subsequent expression. Here again we see the promotion of suppressing components of general empathy to engage in aspects of the skill-based clinical or physician empathy.

We do not disagree with the assertion that the performance of clinical and physician empathy are forms of emotional labor. Nor do we disagree that the persistent enacting of work-appropriate emotional displays or degrees of surface or deep acting deplete psychological and emotional resources. Furthermore, we concur with Vinson and Underman's (2020) underlying assertion that these set of skills associated with clinical and physician empathy are products of socialization and professionalization processes and mechanisms nested within medical education. However, we do argue that the "performance of clinical empathy as emotional labor" stance actually misses the bigger picture, and in fact, a clinically-situated version of empathy, Hojat et al.'s physician empathy in particular, actually promotes an emotion-deficient empathy in the clinical encounter. With the emotional labor perspective, it is important to remember that the emotions in question are not authentic- they are manufactured and produced to be perceived as empathic by the patient. So, while the effort to produce them in order to be perceived as empathic is indeed detrimental (and labor intensive), the process of regulation and production that is clinical and physician empathy may actually necessitate the persistent suppression and suffocation of innate and automatic human capacities related to emotional processing, specifically emotional contagion-which is also labor intensive. Therefore, we argue that this potential suppression of emotional contagion, through the exercise of emotiondeficient clinically-situated empathy, may further exacerbate emotional exhaustion, dehumanization, and the catalysts of burnout. In turn, further research is needed to explore the potentially detrimental physiological consequences of suppressing emotional contagion.

Furthermore, the substantial emphasis on the behavioral component and acting empathetic that is propagated by the clinically-situated version of empathy puts an excessive burden on the observer (i.e. doctor or medical student). In this sense, this behavioral component is somewhat akin to aspects of the emotional labor and surface and deep acting that coincides with clinically-situated empathy-it is producing a behavior, action, sentiment that is perceived as appropriate for the work-based situation. Doing so is not only forcing (or perhaps requiring) a response, which is contrary to general empathy experience, but also converts the doctor-patient encounter 
into little more than a checklist (as per Riess' E.M.P.A.T.H.Y. 2018) where medical students and physicians risk inauthenticity and actually alienating the patient (Wild 2020).

We raise this issue to further emphasize the amount of burden the practice and preaching of a clinically-situated version empathy like physician empathy can put on physicians and medical students - which may, in turn, further deplete psychological, emotional, and physical resources. This is not to say that doctors and medical students should not explicitly acknowledge the patient, cultivate some degree of report with the patient, and show the patient that they understand what they are showing and telling them-but according to classic and contemporary general empathy theories, this is not a necessary step or element of the empathy experience. This manufactured behavioral and/or performative element of the clinically-situated empathy is again, a skill that can be honed and fine-tuned through experience and professional development - but it is not empathy. Taken together, the stakes raised above demonstrate that the embeddedness of an emotion-deficient clinically situated version of empathy not only lends to conceptual and operational confusion within the evergrowing empathy-based research within medical education and healthcare delivery, but also clouds what we are actually assessing among our medical students and healthcare professionals when it comes to "empathy". Moreover, and perhaps most concerning, the propagation of this version of empathy within evaluation, training, and practice may actually be lending to the burnout epidemic among our physician workforce, lending to poorer patient satisfaction reports (due to a lack of perceived authenticity from providers), and perpetuating an emotionally barren healthcare delivery system.

\section{Moving forward}

\section{Theoretical and conceptual expansion}

Empathy scholars in the medical/clinical realm (e.g. Hojat, Halpern, Riess) consistently attempt to extract the cognitive and the emotional components of empathy, but as de Waal (2017) states, "There exists a tendency to treat each aspect separately and dwell on the distinction, but in doing so we lose sight of the functionally integrated whole." Focusing on just the cognitive components individually, allows scholars to cherry-pick what aspects fit standard medical culture, values, and practice. The "high-jacking" of empathy to fit the overarching agenda of the medical institution (Macnaughton 2009) diminishes the value of emotions in the doctor-patient encounter while touting clinical reason, understanding, and cognitive capacity. Yet, the cognitive and emotional components of empathy are not mutually exclusive and do not act independently. The presence of physician socio-emotional challenges notwithstanding, we suggest that clinical and physician empathy scholars return to the conceptualization of general empathy that includes emotional contagion. Given the extensive detail, encompassing conceptualization, theoretical development, and practical evidence, we strongly encourage the utilization of de Waal's Russian Doll 
Model of empathy (de Waal 2008, 2012; de Waal and Preston 2017). The Russian Doll Model presents empathy as a multidimensional, multi-layered concept and experience. The inner core consists of state-matching, motor mimicry, and emotional contagion (what de Waal and others refer to as the perception-action mechanism (PAM)). Built around this hard-wired foundation/core are the outer layers/ shells of the doll that consist of empathic concern and targeting helping. The outermost layers represent cognitive and emotional complex processes of perspectivetaking capacities, self-other distinction, and emotional regulation, but are still fundamentally connected to the PAM core layer.

The Russian Doll model protects the cognitive processes touted by physician empathy, while also spotlighting and integrating the emotional components and explicitly featuring the innate phenomenon of emotional contagion. While spotlighting specific theoretical models may overly simplify complicated and intricate processes and mechanisms, we draw attention to the Russian Doll model here merely to propose a small initial step away from the prevailing and culturally situated framing of emotion-deficient empathy. In short, we are not introducing the Russian Doll model as a final solution, nor are we suggesting consensus over its facticity, but rather spotlighting this inclusive model as a first step in a new direction. Utilizing this encompassing theoretical perspective to discuss, research, and teach empathy reunites doctors and medical students with the human experience of empathy, and unshackles doctors and medical students from the need to experience (and express) a different type of empathy. It may have the potential to reinvigorate humanism within the medical institution, and promotes authenticity, vulnerability, and connectivity while sustaining the value of cognitive processes, understanding, reasoning, and intellect.

Prominent voices in the medical field continue to sound the alarm regarding individual- and structural-level causal factors of burnout, emotional exhaustion, and compassion fatigue among the clinician workforce. Yet, if medical education and practice consistently negate the value of feeling with and interpersonal socioemotional connectivity, and persistently marquee an emotional-deficient version of empathy through research and teaching-then the system is indeed perfectly designed to get the results it gets. Rather, promoting an experience of empathy that acknowledges the social phenomenon of emotional contagion as natural and human not only explicitly and implicitly gives permission to medical students and professionals to feel with, but also pushes back on the requirement of stifling this experience and acting on labor-intensive counterfeit emotions as touted through the physician empathy concept. This would be a fruitful arena for future interdisciplinary research.

\section{Rethinking the conceptualization and operationalization of a clinical skill set}

Given the conflation and conceptual blurring detailed above, we strongly suggest that medicine and medical education scholars not use the term "empathy" when referring to the clinically-situated, emotionally-deficient, primarily cognitive set of interpersonal communicative and behavioral skills (devoid of emotional contagion) 
that represent physician and clinical empathy. Instead, we recommend using terms/ phrases such as Clinical Connectivity, Bedside Behavior, or Clinical Encounter Engagement. These are mere rudimentary, off-the-cuff suggestions-the point being that because "physician empathy" and "clinical empathy" are not empathy and there should be clear conceptual distinction in future research.

Moreover, medicine and medical education scholars could work within the Russian Doll model of empathy while still discussing the skill set associated with a what has become known as a clinically-situated version empathy. In the context of assessment, we are not advocating for the abolishment of Hojat et al.'s physician empathy or the JSPE (or its various offspring). Rather, we suggest that scholars working with the clinical and physician empathy concepts be consistent and explicit with their messaging - that they are measuring a clinical skill set that is related to/associated with, but distinct from general empathy.

As with all research, utilizing a multimethod approach to examine attributes, perceptions, and traits is best-practice, and therefore we encourage future researchers to not only employ other empathy scales alongside the JSPE (e.g. IRI, BEES) but also to engage particular qualitative methods (e.g. observation, interview, focus groups, etc.) if and when feasible in order to better capture the entirety experience of empathy among the sample. Taken a step further, broader ideological and philosophical approaches should be taken to exploring individuals' experience of empathy. Veen et al. (2020) recently suggested that fuzzy concepts that reflect private experiences (i.e. "beetles in boxes") such as empathy, are not only impossible to measure but also impossible to demonstrate. Yet, research consistently shows that "empathic" providers positively affect patient health outcomes and the healthcare industry's bottom line (Decety 2020). Therefore, rather than throw the baby out with the bath water (i.e. not measure or teach empathy), we should gain a better understanding of the social experience of empathy among current and future physicians through more interpretivist- and constructionist-oriented approaches to project design, data gathering, analysis, and presentation by engaging with participatory action research and phenomenology. Currently, the abundant use of the JSPE promotes a standardization of the experience of empathy, one that is devoid of emotion, that may produce "empathy zombies" as Veen et al. would suggest. Exploring the experience of empathy, in any situation, encounter, or interaction, from the perspective of the experiencer is essential to advancing empathy theory-and this requires more qualitative methods such a as interviews, photo-voice, and narrative inquiry, among others.

\section{Teaching empathy}

Recently, there has been an increase in empathy training programs within medical schools (Stepien and Baernstein 2006; Shapiro 2012; Fragkos and Crampton 2020). As we note above, in a recent systematic review of evaluation-based studies of empathy training programs in medical education (Batt-Rawden et al. 2013), 60\% of those that studies that utilized validated outcome measures used the JSPE, with the JSPE used in three times as many studies as the other empathy scales examined. This suggests that much like Sulzer et al. (2016) show that the physician empathy concept 
is embedded in medical education research, Batt-Rawden et al. show that the JSPE (which measures physician empathy specifically) may be somewhat embedded in the empathy training programs within medical education-specifically as it relates to the foundational empathy concept the program was structured around as well as measuring the student-based outcomes of these programs. Yet, as Costa-Drolon et al. (2020) state, empathy-related content can be embedded in various arenas of medical education such as humanities, ethics, narrative medicine, theatre-based, or even theoretical courses and programs, and a thorough and detailed evaluation of where and how empathy is "taught", and/or what type of empathy (i.e. clinicallysituated empathy or general empathy) is "taught" has yet to be conducted. Moreover, Costa-Drolon et al. (2020, p. 6) argue, "The teaching of empathy remains a major unresolved issue, specifically how to teach empathy to medical students in order to ensure empathy in future physicians' practice." Clearly, this would be a fruitful arena for future interdisciplinary research.

From a humanism-in-medicine perspective, we suggest allowing students to "turn into the skid" - embracing emotional contagion as an innate and foundational element of the empathy experience-and work with students and practitioners to hone various empathy muscles to consistently willingly engage in authentic empathy without becoming overwhelmed by others' emotional states. One such program is the Interprofessional Training in Empathy, Affect, and Mindfulness (I-TEAM) (Michalec et al. 2021). Grounded in the more inclusive models of empathy and touting the value of emotional contagion in clinical (and day-to-day) encounters, the I-TEAM Program teaches health professions students emotional state recognition, emotional attunement, accuracy and regulation, self and other awareness, mental flexibility, observation and presence, and listening skills (among others) through shared experiences, empathy workouts, reflection, and mindfulness-based practices.. Rather than focus on empathy as a checklist to complete or a behavior to enact, the I-TEAM program focusing on re-introducing health profession students to the social experience of empathy, celebrating the innate power of emotional contagion (i.e. recognizing it and working with it rather than dismissing and stifling it), and honing their own emotional and cognitive processing capacities to develop what the program leaders refer to as empathic resilience - an ability to experience authentic empathy without becoming overwhelmed or overburdened by others' emotions. Although primarily aims of the I-TEAM program are to enhance students' understanding and perceptions of other health professions (i.e. cultivate interprofessionalism), the pilot program showed significantly positive improvements in empathy and students expressed learning more about empathy as an experience, the social theoretical underpinnings of empathy, and that they could enhance their empathic ability with practices and exercises learned through the program. Given its novelty, it is essential to explore the longitudinal impact of the I-TEAM program (especially as it relates to the focus on emotional contagion) on students' and health professionals' patient engagement as well as their socio-emotional wellbeing. However, we feature the I-TEAM program here to simply note that teaching empathy that is inclusive and promotive of emotional contagion is not only possible within health professions education but can also have positive outcomes for students. 
Taken together, we argue that instituting (through research, practice, and instruction) a broader theoretical, conceptual, and operational approach to empathy within medical education and medical practice that includes key cognitive and emotional aspects-notably emotional contagion-may unlock key elements of authentic interprofessional connectivity that have been suppressed and stifled, and in turn may positivity negate deleterious practices and conditions that facilitate burnout and emotional exhaustion. By focusing more explicitly on the experience of empathy rather than the prominent emotionally-deficient model of clinically-situated empathy, we can potentially reinvigorate the humanistic foundations of patient care, doctor-patient interactions, and what it means to be a healer.

\section{Conclusion}

In this paper we showcased the conceptual differences between empathy in the general sense and the clinically-situated model of empathy. In doing so, we highlighted the innate, involuntary nature of emotional contagion nested within general empathy. We also discussed the embeddedness of Hojat et al.'s physician empathy and how the overarching culture and practices of medicine that problematize emotions and vulnerabilities propagate and sustains this emotion-deficient version of empathy within clinical practice and medical education. We also provided possible consequences to this apparent embeddedness that relate to the burnout and emotional exhaustion epidemic rampant among physicians (and medical students). Alternatively, we argue that employing a more encompassing social theoretical perspective of empathy that includes emotional contagion such as the Russian Doll Model, and utilizing the tenets of this model to not only practicing consistent and direct conceptualization and operationalization in research and commentary, as well as scaffolding for developing future empathy training programs, may facilitate (inter)personal skills, resilience building, and well-being among providers and students.

Acknowledgements The authors would like to acknowledge the Arnold P. Gold Foundation and the Josiah Macy Jr. Foundation for funding projects that led to this theoretical report.

\section{References}

Batt-Rawden, Samantha A., Margaret S. Chisolm, Blair Anton, and Tabor EFlickinger. 2013. Teaching empathy to medical students: An updated Systematic Review. Academic Medicine 88 (8): 1171-1177.

Batson, C.D. 1991. The altruism question: Toward a social-psychological answer. Hillsdale: Lawrence Erlbaum.

Batson, C. Daniel, David A. Lishner, Jennifer Cook, and Stacey Sawyer. 2005. Similarity and nurturance: Two possible sources of empathy for strangers. Basic and Applied Social Psychology. 27 (1): 15-25.

Berman Blix, S. 2019. Different roads to empathy: Stage actors and judges as polar cases. Emotions and Society 1 (2): 163-180.

Brotheridge, Celeste M., and Raymond T. Lee. 2002. Testing a conversation of resources model of the dynamics of emotional labor. Journal of Occupational Health Psychology 7 (1): 57-67. 
Cialdini, Robert B., Mark Schaller, Donald Houlihan, Kevin Arps, Jim Fultz, and Arthur L. Beaman. 1987. Empathy-based helping: Is it selflessly or selfishly motivated? Journal of Personality and Social Psychology 52 (4): 749-758.

Costa-Drolon, Emmanuel, Laurence Verneuil, Emile Manolios, Anne Revah-Levy, and Jordan Sibeoni. 2020. Medical students' perspectives on empathy: A systematic review and metasynthesis. Academic Medicine. https://doi.org/10.1097/ACM.0000000000003655.

Davis, Mark H. 1994. Empathy: A social psychological approach. Boulder, CO. Westview Press.

de Vignegmont, Frederique, and Tania Singer. 2006. The empathic brain: How, when and why? Trends in Cognitive Science 10: 435-441.

de Waal, F. B. M. 2003. In Feelings \& Emotions: The Amsterdam Symposium, ed. Manstead T., Frijda, N. and Fischer A., 379-399. Cambridge Univ. Press.

de Waal, Frans B.M.. 2008. Putting the Altruism back into Altruism: The evolution of empathy. Annual Review of Psychology 59: 279-300.

de Waal, Frans B.M.. 2009. The age of empathy. New York: Harmony Books.

de Waal, Frans B.M.. 2012. The antiquity of empathy. Science 336: 874-876.

de Waal, Frans B.M.., and Stephanie D. Preston. 2017. Mammalian empathy: Behavioural manifestations and neural basis. Nature Reviews Neuroscience 18: 498-509.

Decety, Jean. 2020. Empathy in medicine: What it is, and how much we really need it. The American Journal of Medicine 133 (5): 561-566.

Decety, Jean, and Aikaterini Fotopoulou. 2015. Why empathy has beneficial impact on others in medicine: Unifying theories. Frontiers in Behavioral Neuroscience 8: 1-11.

Decety, Jean, and Philip L. Jackson. 2004. The functional architecture of human empathy. Behavioral and Cognitive Neuroscience Reviews 3 (2): 71-100.

Decety, Jean, and Yoshiya Moriguchi. 2007. The empathic brain and its dysfunction in psychiatric populations: Implications for intervention across different clinical conditions. BioPsychoSocial Medicine 1: 1-22.

Decety, Jean, Chia-Yan. Yang, and Yawei Cheng. 2010. Physicians down-regulate their pain empathy response: An event-related brain potential study. NeuroImage 50: 1676-1682.

Dovidio, John F., Judith L. Allen, and David A. Schroeder. 1990. Specific of empathy-induced helping: Evidence for altruistic motivation. Journal of Personality and Social Psychology 59 (2): 249-260.

Eisenberg, Nancy. 2006. Empathy-related responding and prosocial behavior. In Empathy and fairness, ed. Novartis Foundation. New York: Wiley.

Eisenberg, N., and J. Strayer. 1987. Critical issues in the study of empathy. In Empathy and its development, ed. N. Eisenberg and J. Strayer, 3-15. New York: Cambridge University Press.

Eisenberg, Nancy, Richard A. Fabes, Bridget Murphy, Mariss Karbon, Pat Maszk, Melanie Smith, Cherie O'Boyle, and Karen Suh. 1994. The relations of emotionality and relgulation to dispositional and situational empathy-related responding. Journal of Personality and Social Psychology 66 (4): 776-797.

Eisenberg, Nancy, Richard A. Fabes, and Tracy L. Spinrad. 2006. Prosocial development. In Handbook of Child Psychology Vol. 3: Social, emotional, and personality development, eds. N. Eisenberg, W. Damon, and R. L. Lerner, 646-718. Hoboken, NJ: Wiley.

Feshbach, Norma, Deitch, and Seymour Feshbach. 2009. Empathy and Education. In The Social Neuroscience of Empathy, eds, J. Decety and W. Ickes, 85-97. Cambridge, MA: MIT Press.

Finset, A., and K. Ømes. 2017. Empathy in the clinical-patient relationship: The role of reciprocal adjustments and processes of synchrony. Journal of Patient Experience 4 (2): 64-68.

Fragkos, Konstantinios, and Paul Crampton. 2020. The effectiveness of teaching clinical empathy to medical students: A systematic review and meta-analysis of randomized controlled trials. Academic Medicine 95 (6): 947-957.

Gleichgerrcht, Ezequiel, and Jean Decety. 2012. The costs of empathy among health professionals. In Empathy: From bench to bedside, ed. J. Decety. Cambridge: MIT Press.

Gonzalez-Liencres, Cristina, Georg Juckel, Cumhur Tas, Astrid Friebe, and Martin Brüne. 2014. Emotional contagion in mice: The role of familiarity. Behavioural Brain Research 263: 16-21.

Hafferty, Frederic W., and R. Franks. 1994. The hidden curriculum, ethics teaching, and the structure of medical education. Academic Medicine 69 (11): 861-871.

Halpern, Jodi. 2001. From detached concern to empathy: Humanizing medical practice. New York: Oxford University Press.

Halpern, Jodi. 2003. What is Clinical Empathy? Journal of General Internal Medicine 18: 670-674. 
Halpern, Jodi. 2014. From idealized clinical care to empathic communication in medical care. Medicine, Health Care and Philosophy 17: 301-311.

Hatfield, Elaine, John T. Cacioppo, and Richard L. Rapson. 1993. Emotional contagion. Current Directions in Psychological Science 2 (3): 96-99.

Hatfield, Elaine, John T. Cacioppo, and Richard L. Rapson. 1994. Emotional contagion. New York: Cambridge University Press.

Hochschild, Arlie R. 1983. The managed heart: Commercialization of human feeling. Berkeley: University of California Press.

Hoffman, Martin L. 1981. Is altruism part of human nature? Journal of Personality and Social Psychology 40: 121-137.

Hojat, Mohammadreza. 2007. Empathy in patience care: Antecedents, development, measurement, and outcomes. New York, NY: Springer.

Hojat, Mohammadreza. 2016. Empathy in health professions education and patient care. New York: Springer International.

Hojat, Mohammadreza, Salvatore Mangione, Thomas J. Nasca, Mitchell J.M.. Cohen, Joseph S. Gonnella, James B. Erdmann, J. Veloski, and M. Magee. 2001. The Jefferson Scale of Physician Empathy: Development and preliminary psychometric data. Educational and Psychological Measurement 61 (2): 522-537.

Hojat, Mohammadreza, Joseph S. Gonnella, Salvatore Mangione, Thomas J. Nasca, J. Veloski, James B. Erdmann, C.A. Callahan, and M. Magee. 2002a. Empathy in medical students as related to academic performance, clinical competence and gender. Medical Education 36: 522-527.

Hojat, Mohammadreza, Joseph S. Gonnella, Thomas J. Nasca, Salvatore Mangione, Michael Vergare, and Michael Magee. 2002b. Physician empathy: Definition components, measurement, and relationship to gender and specialty. American Journal of Psychiatry 2002 (159): 1563-1569.

Hojat, Mohammadreza, Michael J. Vergare, Kaye Maxwell, George Brainard, Steven K. Herrine, Gerald A. Isenberg, Jon Veloski, Josephy S. Gonnella. 2009. Academic Medicine 84 (9): 1182-1101.

Hojat, Mohammadreza, Jennifer DeSantis, Stephen C. Shannon, Luke H. Mortensen, Mark R. Speicher, Lynn Bragan, Marianna LaNoue, and Leonard H. Calabrese. 2018. The Jefferson Scale of Empathy: A nationwide study of measurement properties, underlying components, latent variable structures, and national norms in medical students. Advances in Health Sciences Education 23: 899-920.

Hojat, Mohammadreza, Stephen Shannon, Jennifer DeSantis, Mark Speicher, Lynn Bragan, and Leonard Calabrese. 2020. Does empathy decline in the clinical phase of medical education? A nationwide, multi-institutional, cross-sectional study of students at DO-granting medical schools. Academic Medicine 95 (6): 911-918.

Hoffman, M.L. 2000. Empathy and moral development. New York: Cambridge University Press.

Ickes, W. 2003. Everyday mind reading. New York: Prometheus.

Jahoda, Gustav. 2005. Theodor Lipps and the Shift from "sympathy" to "empathy." Journal of the History of the Behavioral Sciences 41 (2): 151-163.

Lanzoni, S. 2018. Empathy: A history. New Haven: Yale University Press.

Larson, Eric B., and Xin Yao. 2005. Clinical empathy as emotional labor in the patient-physician relationship. JAMA 293 (9): 1100-1106.

McNaughton, Nancy. 2013. Discourse(s) of emotion within medical education: The ever-present absence. Medical Education 47 (1): 71-79.

Macnaughton, Jane. 2009. The art of medicine: The dangerous practice of empathy. The Lancet 373 (6): 1940-1941.

McAuliffe, William H.B.., Evan C. Carter, Juliana Berhane, Alexander C. Snihur, and Michael E. McCullough. 2020. Is empathy the default response to suffering? A meta-analytic evaluation of perspective taking's effect on empathic concern. Personality and Social Psychology Review 24 (2): $121-140$.

McCaffree, K. 2020. Towards an integrative sociological theory of empathy. European Journal of Social Theory 23 (4): 550-570.

Meyza, Ksenia, and Ewelina Knapska. 2018. What can rodents teach us about empathy? Current Opinion in Psychology 24: 15-20.

Michalec, B. 2012. The pursuit of medical knowledge and the potential consequences of the hidden curriculum. Health 16 (3): 367. 
Michalec, B., J.M. Schneider, and M. Mackenzie. 2021. Teaching empathy in an interprofessional setting with a focus on decategorization: Introducing I-TEAM. Journal of Interprofessional Education \& Practice 22: 1-11.

Montag, C., and A. Heinz. 2008. Theodor Lipps and the concept of empathy: 1851-1914. American Journal of Psychiatry 165 (10): 1261.

Ofri, Danielle. 2012. Doctors have feelings too. New York Times, March 28, 27.

Osler, W. 1906. Aequanimitas with other addresses to medical students, nurses and practitioners of medicine Philadelphia P”. Philadelphia: Blankiston's Son \& Company.

Pedersen, R. 2009. Empirical research on empathy in medicine: A critical review. Patient Education and Counseling 76 (3): 307-322.

Preston, S.D., and F.B.M. de Waal. 2002. Empathy: Its ultimate and proximate bases. Behavior and Brain Science 25: 1-20.

Preusche, Ingrid, and Claus Lamm. 2016. Reflections on empathy in medical education: What can we learn from social neurosciences? Advances in Health Sciences Education 21: 235-249.

Prochazkova, Eliska, and Mariska E. Kret. 2017. Connecting minds and sharing emotions through mimicry: A neurocognitive model of emotional contagion. Neuroscience and Biobehavioral Reviews 80: 99-114.

Riess, Helen. 2018. The empathy effect. Boulder: Sounds True.

Roberts, William, and Janet Strayer. 1996. "Empathy, Emotional Expressiveness, and Prosocial Behavior." Child Development 67: 449-470.

Ruiz-Junco, N. 2017. Advancing the sociology of empathy: A proposal. Symbolic Interaction 40 (3): 414-435.

Shapiro, J. 2011. Does medical education promote professional alexithymia? A call for attending to the emotions of patients and self in medical training. Academic Medicine 86 (3): 326-332.

Shaprio, J. 2012. The paradox of teaching empathy in medical education. In Empathy: From bench to bedside, ed. J. Decety. Cambridge: MIT Press.

Singer, Tania, and Claus Lamm. 2009. The social neuroscience of empathy. Annals of the New York Academy of Science 1156: 81-96.

Smith, K.D., J.P. Keating, and E. Stotland. 1989. Altruism revisited: The effect of denying feedback on a victim's statusto empathic witness. Journal of Personality and Social Psychology 57: 641-650.

Smither, S. 1977. A reconsideration of the developmental study of empathy. Human Development 20: 253-276.

Stepien, K.A., and A. Baernstein. 2006. Educating for empathy: A review. Journal of General Internal Medicine 21 (5): 524-530.

Sulzer, S.H., N.W. Feinstein, and C. Wendland. 2016. Assessing empathy development in medical education: A systematic review. Medical Education 50 (3): 300-310.

Trevarthen, C. 1979. Communication and cooperation in early infancy: A description of primary intersubjectivity. In Before speech: The beginning of human communication, ed. M. Bullowa, 321-347. New York: Cambridge University Press.

Trevarthen, C., and K.J. Aitken. 2001. Infant intersubjectivity: Theory, and clinical applications. Journal of Child Psychology and Psychiatry 42: 3-48.

Underman, K., and L.E. Hirshfield. 2016. Detached concern? Emotional socialization in twenty-first century medical education. Social Science \& Medicine 160: 94-101.

Veen, M., J. Skelton, and A. de la Croix. 2020. Knowledge, skills and beetles: Respecting the privacy of private experiences in medical education. Perspectives in Medical Education 9: 111-116.

Vinson, Alexandra H., and Kelley Underman. 2020. Clinical empathy as emotional labor in medical work. Social Science in Medicine 251: 1-9.

Wharton, Amy S. 2009. The sociology of emotional labor. Annual Review of Sociology. 35: 147-165.

Wild, Hannah B. 2020. There's no algorithm for empathy. Health Affairs. 39 (2): 339-342.

Wispé, Lauren. 1987. History of the concept of empathy. In Empathy and its Development, ed. N. Eisenberg, J. Strayer, 17-36. New York, NY: Cambridge University Press.

Wispé, Lauren. 1991. The Psychology of Sympathy. New York, NY: Springer.

Publisher's Note Springer Nature remains neutral with regard to jurisdictional claims in published maps and institutional affiliations. 